STRUCTURAL SCIENCE CRYSTAL ENGINEERING MATERIALS

ISSN 2052-5206

\section{Polymorphism in Molecular Crystals. Second Edition. By Joel Bernstein. Oxford University Press, 2020, Pp. 608, Hardcover. Price GBP 85.00. ISBN 9780199655441}

\author{
Claude Lecomte* \\ Universite de Lorraine, CNRS, CRM2, Nancy, France. *Correspondence e-mail: claude.lecomte@univ-lorraine.fr
}

This excellent and very informative book is the second edition of the well known book Polymorphism in Molecular Crystals which the late Professor Bernstein wrote in 2002. This second edition describes major progress in the understanding of polymorphism, from the experimental procedures to show this important property, to applications in fundamental crystallography, structure-properties relationships, material sciences and pharmacology. Many well selected examples are discussed which guide a new researcher into the field. It is also a fantastic source of historical, fundamental and up to date papers and reviews (110 pages of references). This book then can be read by a beginner in the field but also by an active researcher in polymorphism, crystal engineering or crystal chemistry. The author took care to allow the reader to go directly to the information with a well documented index.

Even if it is not defined like that by J. Bernstein, this book is divided into five parts: the first part (chapters 1 to 5) is devoted to fundamentals, analytical and modelling techniques, followed by one general chapter entitled Polymorphism and Structure-Property relations. Then comes the applications to pharmaceuticals, pigments and dyes, highenergy materials. The book ends with the important topic of polymorphism and patents.

Some comments on chapters:

Chapter 2 Fundamentals. This chapter is very chemistry oriented: this is understandable, but some physics about phase transitions could have been useful. Many figures have been reproduced from papers or reviews but sometimes the legends or/and the corresponding text do not help for fully understanding the information of the figure (for example, Figures 2.4 to 2.8 ).

Chapter 3 Exploring the crystal landscape describes the challenge to discover and control the characterizing polymorphism (including disappearing polymorphs) from a thermodynamic and kinetic point of view. Paragraphs on solvent choice, non-traditional techniques and high throughput are especially interesting.

Chapter 4 Analytical Techniques: the most relevant techniques and methods to characterize polymorphs are described: not extensively (it is not the aim of the book) for the usual ones such as X-ray powder or single-crystal diffraction, thermal methods, NMR, IR, Raman spectroscopies, microscopies, but enough to understand which technique should be used for which characterization. The section on optical hot-stage microscopy is very informative. The combination of X-ray diffraction with other methods, such as timeresolved diffraction are also introduced; these new methods (not technologies as written in the book) should have been more developed.

Chapter 5 Computational aspects. First a thorough discussion on conformational polymorphs is given, then methods to calculate $\Delta U$ and $\Delta G$ are proposed with selected examples. The chapter on prediction of polymorphs describes very honestly all blind tests made in collaboration with the Cambridge Structural Database. A large chapter is devoted to Hirshfeld surfaces, which in my opinion, is too long as most information given by Hirshfeld surfaces can be retrieved quantitatively not qualitatively from critical inspection of intermolecular distances and angles. A paragraph on the experimental analysis of the molecular interactions using Bader's Atoms in Molecules theory from high-resolution X-ray diffraction results and/or DFT would have been welcome. 
Chapter 6, Polymorphism and structure-property relations (70 pages) is divided into two parts: bulk physical properties, (electric, optic, mechanic) and molecular properties including photochemical and gas-solid reactions. This chapter which covers all aspects is a strong start to teach a master class in organic crystal physics and physical chemistry.

Chapter 7 discusses polymorphism of pharmaceuticals a subject in itself. Many reviews have already been written on the subject and it is not easy to summarize all the data in 30 pages. This chapter is either to short (for those new to the field) or too long (for crystallographers practising in the field) but gives enough references to the reader. Polymorphism of crystals, but also of co-crystals and amorphous pharmaceuticals are discussed in relation with their biological activity.

Chapters 8 and 9 concern pigments and high-energy materials, an excellent complement to chapter 6 .

Chapter 10 is an important and welcome chapter on polymorphism and patents. Many scientists are not really aware of the world of patents and this chapter guides the reader on how to discuss with lawyers when patents are written. Obviously research on polymorphism will describe more and more new crystal forms and their derived properties, very often protected by new patents. There is today no way to predict solid forms of a given chemical, therefore litigation, especially in the pharmaceutical world is common. In paragraph 10.4 , J. Bernstein discusses in detail some representatives of crystal form patent litigation. Seven selected cases are analysed mostly concerning nature, identity and uniqueness of crystalline modifications.

In conclusion, this second edition of Polymorphism in Molecular Crystals is really welcome; it is clearly written for a broad public with lots of interesting examples. The book covers all applications of polymorphism with a very large selection of references allowing the reader to examine thoroughly any given aspect. 